\title{
Tamoxifeno y antidepresivos: ¿Antagonistas en la prevención del cáncer de mama?
}

\author{
MARÍA ELISA IRARRÁZAVAL O.
}

\section{Antagonism of tamoxifen and antidepressants among women with breast cancer}

Tamoxifen is used as an adjuvant therapy to reduce breast cancer recurrence among women with estrogenreceptor positive tumors. Antidepressants are also commonly used in such women, to treat depression or to manage hot flushes, a frequent tamoxifen secondary effect. Some antidepressants could potentially inhibit cytochrome P450 2D6, required to activate tamoxifen, interfering with its action. Although there is not a clear cut directive on the subject, it is nowadays recommended to treat women with antidepressants with the lower cytochrome P450 2D6 inhibition potential to avoid a possible antagonism that may reduce tamoxifen's prevention of breast cancer recurrence at least in some patients with CYP2D6 genetic variation. The recommended antidepressants are desvenlafaxine, milnacipran, venlafaxin, escitalopram and citalopram.

(Rev Med Chile 2011; 139: 89-99).

Key words: Antidepressive agents; Breast neoplasms; Tamoxifen.

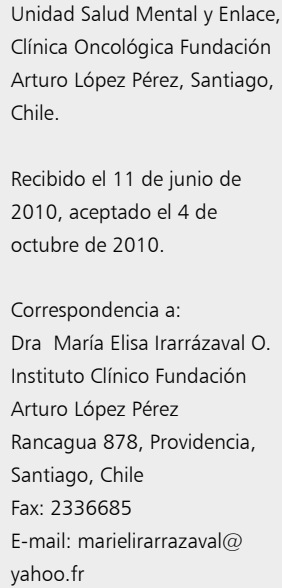

E 1 tamoxifeno (TAM) es un anti estrógeno no esteroidal cuyo uso más frecuente es el tratamiento adyuvante de prevención de recidiva para pacientes pre y post menopáusicas tratadas por cáncer (ca) de mama con receptores estrogénicos (RE) positivos. Ha sido el anti cancerígeno de mayor uso en ca de mama en los últimos 30 años. El endoxifeno es considerado hoy como el metabolito más activo del TAM y existe una gran controversia en relación a las interacciones farmacológicas que puedan existir a nivel de la CYP 2D6, principal enzima responsable de su activación ${ }^{1-7}$.

\section{Mecanismo de acción del tamoxifeno}

Tamoxifeno posee propiedades principalmente antagonistas sobre los RE. Se fija a ellos induciendo un cambio conformacional. Bloquean o alteran así la expresión de los genes que dependen de estos. La mayor parte de la actividad del TAM se observa cuando la célula tumoral se encuentra en la fase G-2 del ciclo, comportándose así como citostático. Además, muestra otros efectos: reduce los niveles del factor de crecimiento insulina símil tipo 1, un factor que estimula la proliferación de las células tumorales e induce la secreción de un factor de transformación del crecimiento que actúa como inhibidor del crecimiento de las células tumorales ${ }^{1,2}$.

\section{Uso clínico y efectividad}

Reduce 45\%-50\% el riesgo de recurrencia del ca de mama. La efectividad del TAM es variable. Se sabe que la expresión de los receptores tumorales estrogénicos y de progesterona influyen en la respuesta clínica. También dependería de los niveles de los diversos metabolitos activos del TAM ${ }^{6,7}$.

\section{Metabolismo}

El tamoxifeno es una prodroga que es transformada en el hígado en varios metabolitos activos. El hidroxitamoxifeno (4-OH tamoxifeno) posee una 
afinidad cien veces mayor por RE que TAM. Ha demostrado mayor potencia pero constituye menos de $10 \%$ de la oxidación primaria de éste. Otro metabolito, el endoxifeno (4 hidroxi-N-desmetil tamoxifeno), posee estas mismas características pero con mayores concentraciones. Se considera hoy el metabolito más activo y algunos estudios han demostrado correlación entre sus niveles séricos y la respuesta clínica. Los principales citocromos involucrados en su metabolismo en estudios in vitro son: CYP3A, CYP2D6, CYP2C9, CYP2C19, CYP2B6 y CYP1A2 (Figura 1). En estudios en vivo destaca la CYP2D6 $6^{3-8}$.

\section{Polimorfismo Genético}

La CYP2D6 se sitúa en el cromosoma 22q13.1. Se han encontrado aproximadamente cien alelos variables, casi todos ellos de actividad reducida o nula comparado con el tipo salvaje o "wild" (wt) (ver actualizaciones en http://www.cypalleles. ki.se $\left.{ }^{9}\right)$. La dotación genética determinará cuatro fenotipos con diversa actividad enzimática, que van desde metabolizadores ultra rápidos (MU), metabolizadores extensos (ME), metabolizadores intermedios (MI), y metabolizadores lentos (ML). Existen numerosos alelos nulos: el más común en caucásicos es el CYP2D $6{ }^{\star} 4$, con una frecuencia de $70 \%$ a $90 \%$. Los ML tendrán mayor probabilidad de experimentar efectos adversos, menores concentraciones de droga activa con potencialmente menos efecto terapéutico y mayor propensión a interacciones con otros fármacos. Diez a quince por ciento de los europeos son MI, y se caracterizan por la alteración de la expresión de la CYP2D6 por los alelos ${ }^{\star} 9,{ }^{\star} 10 y^{\star} 41$. Los ME a su vez constituyen el fenotipo más frecuente de la población europea (60\%-70\%), son el resultado de la presencia de uno o dos alelos con expresión normal (por ej. los alelos ${ }^{\star} 1 \mathrm{y}^{\star} 2$ ), el funcionamiento de la CYP es adecuado. Los MU constituyen $10 \%$ a $15 \%$ de la población caucásica y se caracterizan por presentar duplicaciones genéticas de los alelos normales ${ }^{10-12}$.

En los hispanos estudiados en EEUU se describe una prevalencia de 2,2\% a 6,6\% de ML. Los amerindios tienen una menor incidencia del alelo CYP $2 \mathrm{D}^{\star} 4$, pero los hispanos de origen caucásico tienen una prevalencia de ML similar a la población europea $^{12}$.

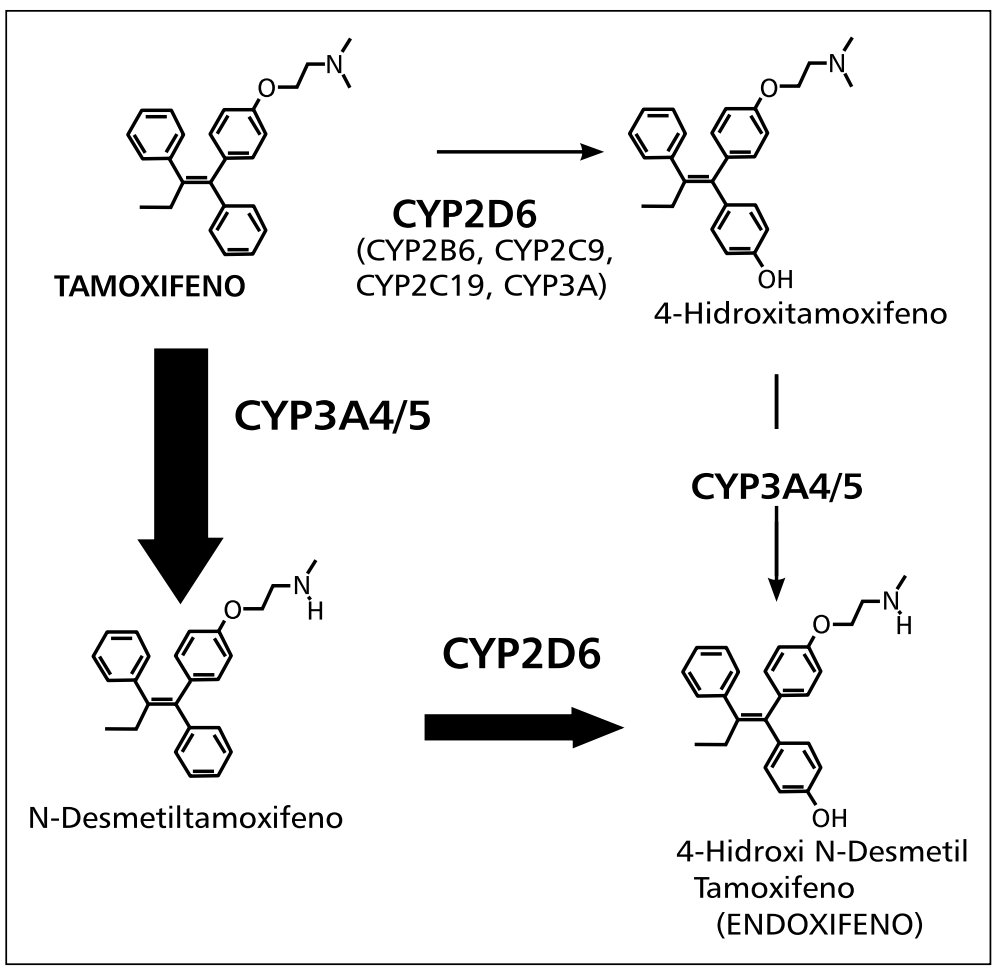

Figura 1. Metabolismo del Tamoxifeno ${ }^{6}$. 


\section{Polimorfismo genético, interacciones farmacológicas y efectividad del TAM}

Goetz et al, estudiaron cambios entre respuesta terapéutica al TAM y variantes de la CYP2D6. En una muestra de un estudio prospectivo fase III de mujeres postmenopáusicas con ca de mama RE (+) en tratamiento con $20 \mathrm{mg} / \mathrm{d}$ de TAM, que investigaba el impacto de la adición del andrógeno fluoximestron por un año, estudió el genotipo de 223 pacientes (alelos ${ }^{\star} 4 \mathrm{y}^{\star} 6$ de las CYP2D6). De las 190 pacientes en que se logró determinar el genotipo del alelo*4, $137(72,1 \%)$ eran $\mathrm{ME}(\mathrm{wt} /$ wt), cuarenta $(21,1 \%) \mathrm{MI}\left(\mathrm{wt} /{ }^{\star} 4\right)$ y trece $(6,8 \%)$ ML $\left({ }^{\star} 4 /{ }^{\star} 4\right)$. Luego de un seguimiento de una media de 11,4 años, los ML se asociaron a un peor pronóstico oncológico. Tuvieron una sobrevida libre de recaída (SLR) y sobrevida libre de enfermedad (SLE) menor. No encontró impacto en la sobrevida global (SG). Dichos resultados fueron confirmados por los mismos autores en una muestra ampliada de 256 pacientes (Tabla 1). Schroth et al, estudiaron de forma retrospectiva una cohorte no randomizada de 206 mujeres con ca de mama postmenopáusicas en tratamiento con TAM, y 280 sin éste. Evaluaron los alelos ${ }^{\star} 4,{ }^{\star} 5,{ }^{\star} 10 \mathrm{y}{ }^{\star} 41$ para cubrir la mayoría de los ML y MI. A una media de seguimiento de 71 meses, las pacientes ML y MI presentaron mayor recurrencia de enfermedad, con una SLE y una SLR menores. Esta asociación no se observó en las pacientes sin tamoxifeno ${ }^{7,13,14}$.

En la población asiática existe una mayor prevalencia de MI por una gran presencia del alelo ${ }^{\star} 10$. Lim et al, incluyeron 202 pacientes con ca de mama primario o metastásico tratadas con $20 \mathrm{mg} / \mathrm{d}$ de TAM por más de ocho semanas. La población estaba constituida por $31,6 \%$ de ME, $44 \%$ de MI $\left(\mathrm{wt} /{ }^{\star} 10\right)$ y $24,2 \%$ de $\mathrm{ML}\left({ }^{\star} 10 /{ }^{\star} 10\right)$. Estas últimas presentaban concentraciones significativamente menores tanto de endoxifeno como de $4-\mathrm{OH}-$ tamoxifeno. En aquellas pacientes con metástasis $(\mathrm{n}=21)$, las seis cuya enfermedad progresó o se mantuvo estable menos de 24 semanas eran ML. Su promedio de tiempo libre de progresión fue significativamente menor $(5,0$ versus 21,8 meses $\mathrm{p}=0,0032$ ). Kiyotani et al, investigaron a $67 \mathrm{pa}-$ cientes tratadas con $20 \mathrm{mg} / \mathrm{d}$ de TAM por cinco años con una media de seguimiento de diez años. Encontraron frecuencias del $29,9 \%$ de $\operatorname{ME}\left({ }^{\star} 1 /{ }^{\star} 1\right)$, $34,3 \%$ de $\mathrm{MI}\left({ }^{\star} 1 /{ }^{\star} 10\right)$ y $22,4 \%$ de $\mathrm{ML}\left({ }^{\star} 10 /{ }^{\star} 10\right)$. Estas últimas tuvieron una mayor incidencia de recurrencia que las ME $(\mathrm{p}=0,0057)$ o MI $(\mathrm{p}=$ 0,0031 ). Estos estudios confirman los hallazgos de la relación entre el genotipo de la CYP2D6 y los resultados del tratamiento del TAM realizados en EEUU y Europa previamente descritos ${ }^{15,16}$.

En pacientes que usan TAM de forma preventiva, se han encontrado resultados similares. En Italia, se evaluó a 5.408 mujeres histerectomizadas entre 35 y 70 años, que fueron aleatoriamente randomizadas para recibir $20 \mathrm{mg} / \mathrm{d}$ de TAM o placebo para prevención de ca de mama. Se estudió a un subgrupo de 46 pacientes que desarrollaron ca de mama y a 136 que no lo hicieron. La frecuencia de CYP2D6 ${ }^{*} 4 /{ }^{\star} 4$ era significativamente más alta en las mujeres que desarrollaron cáncer en el subgrupo asignado a TAM $(\mathrm{p}=0,015)$.

Sin embargo, tres estudios publicados a la fecha no avalan estos hallazgos. Nowell et al estudiaron a 165 pacientes y no encontraron diferencia en la respuesta a TAM entre pacientes con o sin el alelo ${ }^{\star} 4$. En Suecia, se realizó un estudio de 112 pacientes con $40 \mathrm{mg} / \mathrm{d}$ de TAM con una media de 10,7 años de seguimiento. Paradójicamente se encontró una disminución en la recurrencia de la enfermedad en pacientes ML con el alelo CYP2D6 ${ }^{\star} 4 \quad(p=0,0089)$. Este grupo extendió su estudio a 677 mujeres de las cuales 238 fueron randomizadas a dos versus cinco años de tratamiento con TAM. Las mujeres ML $\left({ }^{\star} 4 /{ }^{\star} 4\right)$ tuvieron una SLE significativamente mayor a aquellas $\mathrm{ME}\left({ }^{\star} 1{ }^{\star} 1\right)$ $(\mathrm{p}=0,05)$ o MI $(\mathrm{p}=0,04)^{17-20}$. Algunos reparos hechos a estos resultados son: Esta relación no es significativa en un análisis mutlivariable de Cox $(\mathrm{p}=0,055)$ y las pacientes no estuvieron todas expuestas al mismo tiempo ni dosis de $\mathrm{TAM}^{6}$.

\section{Sustratos, inhibidores e inductores de la CYP2D6}

La CYP 450 2D6 participa en el metabolismo de alrededor de $25 \%$ de los fármacos, incluyendo betabloqueadores, antiarrítmicos, antipsicóticos, antieméticos, opioides, antidepresivos (AD) y la ya mencionada TAM. En la Tabla 2 se muestran sus principales sustratos. La CYP es inducible por rifampicina y dexametasona. Algunos de sus inhibidores son: bupropion, fluoxetina, paroxetina, quinidina, duloxetina, amiodarona, cimetidina, sertralina, clorfenamina, clorpromazina, citalopram, cocaína, doxorrubicina, metoclopramida, metadona, moclobemida, y ranitidina ${ }^{10,11,21}$. 
Tabla 1. Resumen de los principales estudios que evalúan el polimorfismo genético de los metabolizadores del TAM y recurrencia oncológica a largo plazo

\begin{tabular}{|c|c|c|c|}
\hline Referencia & Datos más relevantes & $\begin{array}{l}\text { Inhibidores } \\
\text { CYP2D6 }\end{array}$ & Principales resultados \\
\hline $\begin{array}{l}\text { Goetz et al }{ }^{7} \text {, } \\
\text { año } 2005\end{array}$ & $\begin{array}{l}\text { Prospectivo } \\
223 \text { pacientes con TAM por } 5 \text { años. } \\
\text { Compara } \mathrm{ML}\left({ }^{*} 4{ }^{*} 4\right), \mathrm{n}=13 \text {; con } \mathrm{Ml} \\
\left({ }^{*} 4 / \mathrm{wt}\right), \mathrm{n}=40, \mathrm{y} \mathrm{ME}(\mathrm{wt} / \mathrm{wt}), \mathrm{n}=137 \text {. }\end{array}$ & No evalúa & $\begin{array}{l}\text { 1. Menor tiempo libre de recidiva } \\
\text { ( } p=0,023) \text { y sobrevida libre de enfer- } \\
\text { medad }(p=0,012) \text { en } M L \text { que los otros } \\
\text { genotipos } \\
\text { 2. No observa diferencia en sobrevida } \\
\text { global ( } p=0,169) \text { comparado con los } \\
\text { otros dos grupos }\end{array}$ \\
\hline $\begin{array}{l}\text { Goetz et al13, } \\
\text { año } 2007\end{array}$ & $\begin{array}{l}\text { Prospectivo } \\
256 \text { pacientes con TAM por } 5 \text { años. } \\
\text { Compara: Grupo I: al menos un alelo } \\
\text { *4 o/y uso de inhibidor potente de la } \\
\text { CYP }(n=16) \text { contra Grupo II: ML o } \\
\text { MI sin inhibidores de la CYP o/y uso de } \\
\text { inhibidores moderados }(n=155)\end{array}$ & Sí evalúa & $\begin{array}{l}\text { 1. Sobrevida libre de recidiva menor en } \\
\text { Grupo I }(p=0,034) \text { y tendencia a menor } \\
\text { sobrevida global }(p=0,083) \text { en relación } \\
\text { al Grupo II }\end{array}$ \\
\hline $\begin{array}{l}\text { Schroth et } \mathrm{al}^{14} \text {, } \\
\text { año } 2007\end{array}$ & $\begin{array}{l}\text { Retrospectivo } \\
206 \text { pacientes con TAM } \\
\text { Compara ML+MI: portadores de *4, *5, } \\
* 10, * 41(n=30) \text { con ME }(n=167)\end{array}$ & No evalúa & $\begin{array}{l}\text { 1. Mayor riesgo de recurrencia ( } p= \\
0,02) \text { en } \mathrm{Ml}+\mathrm{Ml} \text { comparados con } \mathrm{ME} \\
\text { 2. Tendencia a menor sobrevida global } \\
\text { en } \mathrm{ML}+\mathrm{MI}(\mathrm{p}=0,11) \text { que en } \mathrm{ME}\end{array}$ \\
\hline $\begin{array}{l}\text { Lim et al }{ }^{15} \text {, } \\
\text { año } 2007\end{array}$ & $\begin{array}{l}\text { Prospectivo } \\
202 \text { pacientes con TAM por al menos } \\
8 \text { semanas. } \\
\text { Compara } \mathrm{ML}\left({ }^{*} 10 /{ }^{*} 10\right), \mathrm{n}=49 \text {, con } \\
\text { otros genotipos }(\mathrm{n}=153)\end{array}$ & No evalúa & $\begin{array}{l}\text { 1. Menor concentración de endoxifeno,e } \\
\text { hidroxi-tamoxifeno en sangre. } \\
\text { 2. Sobrevida libre de progresión de la en- } \\
\text { fermedad menor en ML: } 5 \text { meses versus } \\
21,8 \text { meses con otros genotipos } \\
(p=0,0032) \text {. }\end{array}$ \\
\hline $\begin{array}{l}\text { Kiyotani et al }{ }^{16} \text {, } \\
\text { año } 2008\end{array}$ & $\begin{array}{l}\text { Retrospectivo } \\
67 \text { pacientes con TAM. } \\
\text { Compara } \mathrm{ML}\left({ }^{*} 10 /{ }^{*} 10\right) ; \mathrm{n}=15, \text { versus } \\
\mathrm{ME}\left({ }^{*} 1 /{ }^{*} 1\right), \mathrm{n}=20 \text { o } \mathrm{MI}\left({ }^{*} 1 /{ }^{*} 10\right), \mathrm{n} \\
=23\end{array}$ & $\begin{array}{c}\text { Descarta uso } \\
\text { de IRSS }\end{array}$ & $\begin{array}{l}\text { 1. Mayor riesgo de recurrencia en } \mathrm{ML} \\
(\mathrm{p}=0,0057) \text { a los } 10 \text { años que } \mathrm{ME} \text { o Ml } \\
(\mathrm{p}=0,0079)\end{array}$ \\
\hline $\begin{array}{l}\text { Bonanni et al17, } \\
\text { año } 2006\end{array}$ & $\begin{array}{l}\text { Prospectivo } 46 \text { mujeres de alto riesgo } \\
\text { de Ca de Mama con TAM preventivo } \\
20 \mathrm{mg} / \mathrm{d} \text {, compara ML }\left(* 4 /{ }^{*} 4\right), \mathrm{n}=3 \text {, } \\
\text { con ME (wt/wt), n=13, y Ml ( wt/*4), } \\
n=4\end{array}$ & No evalúa & $\begin{array}{l}\text { Mayor riesgo de cáncer en } \mathrm{ML} \text { versus } \\
\text { otros genotipos }(8,7 \% \text { versus } 0,7 \%)\end{array}$ \\
\hline $\begin{array}{l}\text { Nowell et } a^{18} \text {, } \\
\text { año } 2005\end{array}$ & $\begin{array}{l}\text { Retrospectivo } \\
166 \text { pacientes con TAM adjuvante, } \\
\text { compara ME (wt/wt), } \mathrm{n}=114 \text {, con } \mathrm{ML} \\
\left({ }^{*} 4 /{ }^{*} 4\right)+\mathrm{Ml}\left({ }^{*} 4 / \mathrm{wt}\right), \mathrm{n}=48\end{array}$ & No evalúa & $\begin{array}{l}\text { No encuentra asociación entre recu- } \\
\text { rrencia de enfermedad }(p=0,67) \circ \\
\text { sobrevida libre de enfermedad }(p=0,19) \\
\text { y genotipo CYP2D6. }\end{array}$ \\
\hline $\begin{array}{l}\text { Wegman et al }{ }^{19}, \\
\text { año } 2004\end{array}$ & $\begin{array}{l}\text { Retrospectivo } \\
112 \text { pacientes con TAM por } 2 \text { años, } \\
\text { Compara } \mathrm{ML} \text { o } \mathrm{MI}\left({ }^{*} 4 /^{*} 4 \text { o } 4 / /^{*} 1\right) \text {, } \\
n=24 \text {, con } \mathrm{ME}\left(* 1 /{ }^{*} 1\right) ; n=52 \text {. }\end{array}$ & No evalúa & $\begin{array}{l}\text { Sobrevida libre de enfermedad MAYOR } \\
\text { en } M L \text { o MI versus ME }(p=0,14)\end{array}$ \\
\hline $\begin{array}{l}\text { Wegman et } a^{20}, \\
\text { año } 2005\end{array}$ & $\begin{array}{l}\text { Retrospectivo } \\
238 \text { pacientes con TAM, } 20 \text { o } 40 \mathrm{mg} / \mathrm{d} \\
\text { por } 2 \text { ó } 5 \text { años } \\
\text { Compara } \mathrm{ME}\left(* 1 /{ }^{*} 1\right), \mathrm{n}=151 \text {, con } \\
\mathrm{Ml} \circ \mathrm{ML}\left({ }^{*} 1 /{ }^{*} 4 \mathrm{o}^{*} 4 /{ }^{*} 4\right), \mathrm{n}=63\end{array}$ & No evalúa & $\begin{array}{l}\text { Mayor sobrevida libre de enfermedad en } \\
M L \text { y } M I \text { versus } M E(p=0,05)\end{array}$ \\
\hline
\end{tabular}

ML: metabolizadores lentos, MI: metabolizadores intermedios, ME: metabolizadores extensos; TAM: tamoxifeno; wt: wild tipe o alelo salvaje. 
Tabla 2. Sustratos de la CYP450 2D6

\begin{tabular}{|ccll|}
\hline Beta bloqueadores & Antidepresivos & Neurolépticos & Otros \\
S-metoprolol & Tricíclicos & Haloperidol & Anfetamina \\
\hline Propafenona & Paroxetina & Perfenazina & Atomoxetina \\
\hline Timolol & Fluoxetina & Risperidona & Metoxianfetamina \\
\hline Propanolol & Sertralina & Tioridacina & Tramadol \\
& Mirtazapina & aripiprazol & Amiodarona \\
& Fluvoxamina & Zuclopentixol & Codeina \\
& Escitalopram & Clorpromacina & Cimetidina \\
& Duloxetina & & Lidocaina \\
& Venlafaxina & & Tamoxifeno \\
\hline
\end{tabular}

\section{Efectos adversos del tamoxifeno}

El TAM presenta varios efectos adversos. Los más frecuentes son los síntomas de tipo menopáusico (bochornos, síntomas menstruales, sudores, náusea, etc) que se presentan hasta en un 60\%$70 \%$ de los casos. Estos las llevan con frecuencia a discontinuar su tratamiento ${ }^{22,23}$.

\section{Depresión y uso de antidepresivos en Ca de mama}

La depresión en pacientes con ca de mama es más frecuente que en la población general. La prevalencia encontrada es de $25 \%$ a $50 \%$. El diagnóstico en ellas debe centrarse en los síntomas psíquicos como tristeza, anhedonia, desesperanza, sentimientos de culpa, pensamientos de muerte o ideación suicida recurrentes, irritabilidad, agresividad y aislamiento social. Los síntomas físicos o cognitivos como la disminución de peso, la anergia, la lentitud física o cognitiva, el insomnio, etc, suelen ser consecuencia de la misma enfermedad o efectos secundarios de los diversos tratamientos. Existen varios casos publicados que destacan al TAM como desencadenante de depresión lo cual no se ha podido demostrar en estudios más grandes ${ }^{24-26}$.

En relación al tratamiento, los $\mathrm{AD}$ más frecuentemente indicados en estas pacientes son los inhibidores de la recaptación de serotonina, tricíclicos (especialmente la amitriptilina) y los inhibidores de la recaptación de serotonina y noradrenalina ${ }^{24}$.
Los $\mathrm{AD}$ también se han demostrado útiles en el tratamiento de los bochornos. La paroxetina y la venlafaxina se han usado extensamente para este tratamiento primero en paciente postmenopáusicas y luego en pacientes oncológicas con excelentes resultados. Otros estudios se han realizado con citalopram, sertralina y fluoxetina con menores resultados clínicos ${ }^{28-33}$.

\section{Antidepresivos y tamoxifeno}

La gran mayoría de los $\mathrm{AD}$ son metabolizados, al menos parcialmente, por la CYP2D6 y muchos inhiben su actividad enzimática. Esto ha sido estudiado extensamente in vitro pero en vivo, al igual que para el TAM, influye además el polimorfismo genético de cada paciente lo cual ha dificultado su estudio $^{10,11,34}$.

En estudios in vitro, se ha demostrado que los $\mathrm{AD}$ tricíclicos tienen rutas farmacológicas similares y complejas con participación variable de la CYP2D6 tanto en la formación de metabolitos activos como en su clearence. En cambio, paroxetina, fluoxetina y sus metabolitos son potentes inhibidores de la CYP2D6. Fluvoxamina, citalopram y sertralina son inhibidores intermedios, y venlafaxina y mirtazapina lo son menos. Desvenlafaxina y milnacipran no interactúan. Pese a que bupropión no es un fuerte inhibidor, sí lo son sus principales metabolitos. Estudios en vivo en relación a interacciones con otros fármacos que también se metabolizan en la CYP 2D6, han demostrado que fluoxetina, paroxetina y bupropión 
interfieren en el metabolismo de otras drogas, lo cual no se demostró con venlafaxina, desvenlafaxina ni mirtazapina. Con citalopram, escitalopram, sertralina y duloxetina, se demostró aumento de los sustratos de la CYP, demostrándose cierto grado de inhibición de ésta ${ }^{11}$.

La muy frecuente combinación farmacológica TAM-AD y sus interacciones farmacológicas potencialmente antagónicas a nivel de la CYP 2D6 han impulsado el diseño de diversos estudios para evaluarla.

\section{Polimorfismo genético, interacciones farmacológicas y niveles de endoxifeno en sangre}

Los primeros estudios demostraron en su mayoría, que el metabolismo de TAM se veía francamente afectado por el polimorfismo genético y la administración de fármacos inhibidores de la CYP2D6 (Tabla 3). Jin et al encontraron que mujeres con uno o dos alelos nulos de la CYP2D6 (ML o MI) tenían concentraciones menores de endoxifeno que las pacientes ME. Además, en todas las pacientes que tomaban fármacos que inhibían fuertemente dicha CYP (paroxetina, fluoextina, sertralina, citalopram, amiodarona y metocloparmida), se encontró una menor concentración de endoxifeno en sangre que sus pares que no tomaban inhibidores. Por ejemplo, en mujeres $\mathrm{ME}$ con uso de inhibidores, la concentación de endoxifeno fue $58 \%$ más baja que las que no tomaban dicha medicación. Compararon además el uso de venlafaxina, sertralina y paroxetina y encontraron que venlafaxina bajaba menos los niveles de endoxifeno que los otros dos AD. Este estudio se amplió a 130 pacientes y se estudiaron 27 alelos de la CYP encontrándose los mismos resultados ${ }^{35-39}$.

Desde entonces se hace necesario evaluar no sólo el genotipo de las pacientes, sino también su modulación por otros fármacos para considerar el fenotipo final como se muestra en la Tabla $4^{13}$.

\section{Polimorfismo genético, interacciones farmacológicas con $\mathrm{AD}$ y efectividad del tamoxifeno}

Existen a la fecha pocos estudios sobre polimorfismo genético, uso de inhibidores de la CYP2D6 y efectividad clínica del TAM. Lehmann et al realizaron un estudio caso control $(\mathrm{n}=28)$ para evaluar la exposición a inhibidores de la CYP2D6 (fluoxetina, paroxetina y sertralina) en pacientes con recurrencia de ca de mama y sin ella y no encontraron diferencias entre pacientes que usaron o no dichos inhibidores, pero el polimorfismo genético no fue evaluado y la muestra fue pequeña. Chubak et al, realizaron un estudio retrospectivo de una cohorte de 1306 pacientes con o sin TAM, revisando en las fichas clínicas el uso de $\mathrm{AD}$. Un tercio de las pacientes usó $\mathrm{AD}$ (Inhibidor de recaptación de serotonina o tricíclico), pero no encontraron asociación entre riesgo de recurrencia y uso de $\mathrm{AD}$ en general ni por tipo específico de AD. En pacientes en tratamiento con TAM y uso de de fluoxetina o paroxetina encontraron un leve aumento del riesgo de recurrencia pero el tamaño de la muestra no permitió concluir. Tampoco evaluaron el polimorfismo genético. Lash et al realizaron un estudio retrospectivo de 184 pacientes con ca de mama recidivado versus 184 sin recidiva, $9 \%$ del primer grupo y $11 \%$ del segundo usaron citalopram. No hallaron diferencias en el riesgo de recurrencia entre ambos grupos ${ }^{40-42}$.

Un estudio retrospectivo de Goetz et $\mathrm{al}^{13}$, evaluó la respuesta clínica en pacientes menopáusicas tratadas por ca de mama con TAM según su polimorfismo y la presencia de inhibidores de la CYP2D6. Estos fueron: inhibidores potentes: fluoxetina y paroxetina; y moderados: sertralina, cimetidina, amiodarona, doxepina, ticlopidina y haloperidol. Se comparó tres grupos de pacientes divididos en: Grupo 1: ME sin ningún inhibidor de la CYP2D6; Grupo 2: ME con algún inhibidor o MI sin Inhibidor y Grupo 3: MI con inhibidor o ML. Se midió: Tiempo estimado de progresión, sobrevida libre de recurrencia, SLE y SG. Se encontró una diferencia significativa en los tres primeros indicadores pero no se encontró diferencia significativa en SG. Se concluyó que el metabolismo de la CYP2D6 pareciera ser un predictor pronóstico independiente en las mujeres con ca de mama que reciben TAM, y que los inhibidores de las CYP2D6 debieran evitarse en dichas pacientes. Algunas críticas a dicho trabajo son: muestra pequeña, población homogénea y una determinación muy reducida de alelos de la CYP2D6 ${ }^{6,13}$. Finalmente, dos "abstracts" fueron presentados a la ASCO el 2009 en relación a uso de inhibidores de la TAM y resultados clínicos en mujeres en tratamiento con dicho fármaco. El primero, un estudio fármaco 
Tabla 3. Resumen de los principales estudios que evalúan el polimorfismo genético de los metabolizadores del tamoxifeno y uso de antidepresivos

\begin{tabular}{|c|c|c|c|c|}
\hline Referencia & $\begin{array}{l}\text { Datos más } \\
\text { relevantes }\end{array}$ & $\begin{array}{l}\text { Niveles } \\
\text { de TAM }\end{array}$ & Uso de AD & Principales resultados \\
\hline $\begin{array}{l}\text { Sterns et al }{ }^{35} \text {, } \\
\text { año } 2003\end{array}$ & $\begin{array}{l}\text { Prospectivo, abierto, } \\
\text { observacional. } \\
\text { Mujeres con TAM } \\
\text { adyuvante, } \mathrm{n}=12 \text { : } \\
7 \text { ME y } 5 \mathrm{Ml}\end{array}$ & $\begin{array}{l}\text { Niveles de TAM } \\
\text { y metab-olitos } \\
\text { pre y } 4 \text { semanas } \\
\text { post } 10 \mathrm{mg} / \mathrm{d} \\
\text { de paroxetina }\end{array}$ & $\begin{array}{l}\text { SI, } \\
\text { paroxetina. }\end{array}$ & $\begin{array}{l}\text { 1. Niveles de } E \text { en plasma más bajos en } M I \\
\text { que en } M E(p=0,002) \text { pre paroxetina } \\
\text { 2. Disminución de niveles de } E \text { en todas las } \\
\text { pacientes desde } 2,4 \text { a } 5,5 \mathrm{ng} / \mathrm{mL} \text { (promedio) } \\
\text { post paroxetina }(p=0,004) \\
\text { 3. En } \mathrm{ME} \text {, mayor disminución de la concen- } \\
\text { tración de } \mathrm{E} \text { (de } 64 \% \text { ) que en } \mathrm{Ml}(24 \%) \text { ( } \mathrm{p}= \\
0,03 \text { ) }\end{array}$ \\
\hline $\begin{array}{l}\text { Jin et } a^{36}, \\
\text { año } 2005\end{array}$ & $\begin{array}{l}\text { Prospectivo: } 78 \\
\text { Mujeres con TAM, } \\
\text { Compara } 47 \mathrm{ME} \\
\text { con28 } \mathrm{Ml} \text { y } 3 \mathrm{ML}\end{array}$ & $\begin{array}{l}\text { Niveles de TAM } \\
\text { y metabolitos } \\
\text { antes y } 4 \text { meses } \\
\text { post administra- } \\
\text { ción de } A D\end{array}$ & $\begin{array}{l}\text { SI, } \\
\text { paroxetina, } \\
\text { sertralina, y } \\
\text { venlafaxina }\end{array}$ & $\begin{array}{l}\text { 1. Concentraciones de } \mathrm{E} \text { significativamen- } \\
\text { te menores en } \mathrm{ML}(\mathrm{p}=0,003) \text { y } \mathrm{MI}(\mathrm{p}= \\
0,003 \text { ) comparados con } \mathrm{ME} \\
\text { 2. Concentraciones de TAM, y } \mathrm{H} \text { no signifi- } \\
\text { cativamente diferentes entre los grupos ( } \mathrm{p}= \\
0,92 ; \mathrm{p}=0,86 \text { respectivamente) } \\
\text { 3. Con paroxetina: Concentración de E pro- } \\
\text { medio menor en } 6 \mathrm{ME} \text { con paroxetina, com- } \\
\text { parada con } 34 \text { controles ME sin paroxetina } \\
\text { 4. Con sertralina: Concentración de } \mathrm{E} \text { inter- } \\
\text { medio, en } 4 \mathrm{ME} \text { con sertralina, comparado } \\
\text { con los pacientes con paroxetina y los con } \\
\text { venlafaxina } \\
5 \text {. Con venlafaxina: Prácticamente sin cam- } \\
\text { bio en la concentración de } \mathrm{E}(2 \mathrm{ME} \text { y } 1 \mathrm{MI})\end{array}$ \\
\hline $\begin{array}{l}\text { Borges et } a^{39}, \\
\text { año } 2006\end{array}$ & $\begin{array}{l}\text { Prospectivo, } 158 \text { pa- } \\
\text { cientes con TAM, pre } \\
\text { y postmenopáusicas, } \\
\mathrm{n}=60 \text { con genoti- } \\
\text { po, } 53 \mathrm{ME} \text { y } 7 \mathrm{ML}\end{array}$ & $\begin{array}{l}\text { Niveles de TAM } \\
\text { y metabolitos a } \\
\text { los } 4 \text { meses. }\end{array}$ & $\begin{array}{l}10 \text { ME con } \\
\text { citalopram } \\
\text { o sertralina } \\
5 \text { ME con } \\
\text { fluoxetina o } \\
\text { paroxetina } \\
3 \text { ME con } \\
\text { venlafaxina } \\
\mathrm{n}=6\end{array}$ & $\begin{array}{l}\text { 1. No encuentra diferencia en las concen- } \\
\text { traciones de } T A M \text { ni de } H \text { entre pacientes } \\
\text { con o sin } A D \\
\text { 2. Disminución de las concentraciones de } E \\
\text { en pacientes con } A D(p<0,01) \\
\text { 3. Con citalopram o sertralina: tendencia } \\
\text { hacia menores concentraciones de } E \text { com- } \\
\text { paradas con ME sin inhibidores } \\
\text { 4. Con fluoextina o paroxetina: Marcada } \\
\text { disminución de niveles de endoxifeno }(23,5 \\
\pm 9,5 \text { nmol/L) comparadas con ME sin inhi- } \\
\text { bidores ( } 84,1 \pm 39,4 \text { nmol/L; } p<0,0001) \\
5 \text {. Con venlafaxina: Sin cambios significati- } \\
\text { vos en las concentraciones plasmáticas de } \\
\text { endoxifeno }\end{array}$ \\
\hline $\begin{array}{l}\text { Goetz et } a^{13}{ }^{13} \\
\text { año } 2007\end{array}$ & $\begin{array}{l}\text { Prospectivo, } 256 \\
\text { pacientes con TAM } \\
\text { por } 5 \text { años. Compa- } \\
\text { ra: Grupo I: al menos } \\
\text { un alelo *4 y/o uso } \\
\text { de inhibidor potente } \\
\text { de la CYP ( } n=16) \\
\text { contra Grupo II: ML } \\
\text { o Ml sin inhibidores } \\
\text { de la CYP o/y uso de } \\
\text { inhibidores modera- } \\
\text { dos }(n=155)\end{array}$ & NO & SI & $\begin{array}{l}\text { Grupo I menor sobrevida libre de recurrencia } \\
(p=0,005) \text { y tendencia a menor sobrevida } \\
\text { global }(p=0,077) \text { que el Grupo } \|\end{array}$ \\
\hline
\end{tabular}

E: Endoxifeno, TAM: Tamoxifeno, H: Hidroxitamoxifeno, AD: antidepresivos, ME: metabolizadores extensos, MI: metabolizadores intermedios; ML: metabolizadores lentos. 
Tabla 4. Clasificación de los fenotipos de la CYP 2D6 y su modulación ${ }^{3}$

\begin{tabular}{|c|c|c|}
\hline Tipo de metabolizador & $\begin{array}{l}\text { Actividad CYP } \\
\text { enzimática }\end{array}$ & $\begin{array}{l}\text { Fenotipo uso concomitante de inhibidores de la } \\
\text { CYP2D6: }\end{array}$ \\
\hline Metabolizador lento & Sin actividad & $\begin{array}{l}\text { Dos alelos nulos }\left(\mathrm{CYPD}^{*} 3, * 4, * 5, * 6, * 11\right) \text {, o un alelo nulo con } \\
\text { inhibidor potente }\end{array}$ \\
\hline Metabolizador intermedio & Actividad reducida & $\begin{array}{l}\text { Un alelo nulo (CYP2D6*3,*4,*5,*6, *11) o uno o dos variantes } \\
\text { de los alelos: }{ }^{*} 9, * 10, * 17,{ }^{*} 37, * 41 ; \text { y/o un inhibidor moderado }\end{array}$ \\
\hline Metabolizador extenso & Actividad normal & Dos alelos salvajes (CYP2D6*1, $\left.{ }^{*} 2,{ }^{*} 35\right)$ y sin inhibidor \\
\hline Metabolizador ultra rápido & Actividad Excesiva & Alelos salvajes (CYP2D6*1, *2, *35) duplicados y sin inhibidor \\
\hline
\end{tabular}

Tabla 5. Riesgo de interacción con TAM de antidepresivos según metabolismo en CYP2D611,36

\begin{tabular}{|cllc|}
\hline Interacción mínima & \multicolumn{1}{c}{ Leve } & Moderada & Desaconsejados \\
Desvenlafaxina & Escitalopram & Sertralina* & Paroxetina* \\
Venlafaxina* & Citalopram & Duloxetina & Fluoxetina* \\
Milnacipram & & Fluvoxamina & Bupropión \\
Mirtazapina & & & \\
\hline
\end{tabular}

*Con estudios clínicos con Tamoxifeno.

epidemiológico retrospectivo en 1990 mujeres en tratamiento con TAM, no encontró correlación entre uso de inhibidores de la TAM y recurrencia de la enfermedad. El segundo revisó bases de datos de centros médicos y expendios de farmacias de inhibidores del TAM e investigó el riesgo de recurrencia de ca de mama en pacientes que además usaron inhibidores moderados o potentes de la CYP2D6. Encontraron un riesgo 1,9 veces mayor de recurrencia de ca de mama en pacientes que usaron inhibidores ${ }^{44,45}$.

Un resumen de la evidencia de interacciones TAM-AD se encuentra en la Tabla 5.

Se ha planteado estudiar el genotipo de las pacientes antes de indicar tratamiento $\mathrm{AD}$ a pacientes con TAM. Sin embargo, la ASCO 2007, decidió no implementar aún esta medida por falta de evidencias concluyentes sobre la interacción TAM-AD ${ }^{46-48}$.

\section{Otras interrogantes}

La CYP2D6 podría no ser el único factor responsable en la relación TAM-AD-respuesta clínica. Algunos autores plantean que otras enzimas pudiesen cobrar importancia en los niveles de endoxifeno en sangre una vez inhibida la CYP2D6, como la CYP3A u otras que participan en el clearence del endoxifeno. La respuesta clínica al TAM pudiese también relacionarse con otras enzimas que participan de la farmacodinamia del TAM, como por ejemplo las relacionadas a los genes del tumor mismo responsables de la resistencia a estrógenos y aquellos asociados a la proliferación celular mediada por los RE. Estos factores no han sido tomados en cuenta en los estudios publicados sobre este tema. Más conocimiento al respecto, permitiría entender mejor la variabilidad interindividual de la respuesta a TAM y su interacción con los $\mathrm{AD}^{39,46-48}$.

\section{Conclusiones}

La elección del $\mathrm{AD}$ a indicar en pacientes con ca de mama debe tomar en cuenta, según la clínica: su perfil ya sea activante, ya sea sedante; sus efectos secundarios y el menor potencial de interacción medicamentoso posible ${ }^{26}$.

No podemos concluir aún que exista una interacción peligrosa entre antidepresivos y TAM. No obstante, hay bastante evidencia científica que apoya esta tesis especialmente en pacientes 
MI y ML. Se ha demostrado que en ellos se produce una inhibición de la CYP2D6 con menores niveles de metabolitos activos de TAM en sangre. Hay poca evidencia aún en relación a una menor protección a una recidiva del ca de mama. Hasta tener más información, recomendamos evitar el uso de fluoxetina, paroxetina y bupropión, ya sea como tratamiento antidepresivo, ya sea para el tratamiento de bochornos. Los fármacos recomendados son: desvenlafaxina y milnacipran, venlafaxina, escitalopram, y citalopram. Mirtazapina es un excelente antidepresivo que no interactúa con el CYP pero el aumento de peso que suele provocar, representa otro factor de riesgo de recidiva a evitar en esta población ${ }^{49,50}$. Sertralina, disponible en el Sistema Nacional de Salud chileno, puede ser utilizada de no contarse con los fármacos anteriormente descritos ya que posee cierto potencial inhibitorio de la CYP.

Finalmente, faltan estudios con un número de pacientes adecuados que correlacionen polimorfismo genético, niveles de endoxifeno (y otros metabolitos activos), uso de antidepresivos (y de otros inhibidores de la CYP2D6), y resultados clínicos oncológicos para una orientación definitiva en el uso de $\mathrm{AD}$ en pacientes con TAM.

\section{Referencias}

1. Early Breast Cancer Trialists' Collaborative Group. Tamoxifen for early breast cancer: an overview of the randomised trials. Lancet 1998; 351 (9114): 1451-67.

2. Early Breast Cancer Trialists' Collaborative Group.Effects of chemotherapy and hormonal therapy for early breast cancer on recurrence and 15-year survival: an overview of the randomised trials. Lancet 2005; 365 (9472): 1687-717.

3. Johnson MD, Zuo H, Lee KH, Trebley JP, Rae JM, Weatherman RV, et al. Pharmacological characterization of 4-hydroxy-N-desmethyl tamoxifen, a novel active metabolite of tamoxifen. Breast Cancer Res Treat 2004; 85: 151-9.

4. Desta Z, Ward BA, Soukhova NV, Flockhart DA. Biotransformation by the Human Cytochrome P450 System in Vitro: Prominent Roles for CYP3A and CYP2D6. J Pharmacol Exp Ther, 2004; 310: 1062-75.

5. Lim Yc, Desta Z, Flockhart DA, Skaar TC. Endoxifen (4-hydroxy-N-desmethyl-tamoxifen) has anti-estrogenic effects in breast cancer cells with potency similar to 4-hydroxytamoxifen. Cancer Chemother Pharmacol
2005; 55: 471-8.

6. Stearns V, Rae JM. Pharmacogenetics and breast cancer endocrine therapy: CYP2D6 as a predictive factor for tamoxifen metabolism and drug response?. Expert Reviews in Molecular Medicine 2008; Cambridge University Press 2008 , disponible en http://www.expertreviews. org/ (consultado en noviembre 2009).

7. Goetz MP, Rae JM, Suman VJ, Safgren SL, Ames M, Visscher DW, et al. Pharmacogenetics of tamoxifen biotransformation is associated with clinical outcomes of efficacy and hot flashes. J Clin Oncol 2005; 23: 3708-9.

8. Beverage JN, Sissung TM, Sion AM, Danesi R, Figg WD. CYP2D6 polymorphisms and the impact on tamoxifen therapy. J Pharm Sci 2007; 96: 2224-31.

9. Allele Nomenclature Committee. Home Page of the Human Cytochrome P450 (CYP) en http://www.cypalleles. ki.se. (consultado en marzo 2010).

10. Shu-Feng Zhou P. Polymorphism of Human Cytochrome P450 2D6 and Its Clinical Significance Part I. Clin Pharmacokinet 2009; 48: 689-723.

11. Shu-Feng Zhou P. Polymorphism of Human Cytochrome P450 2D6 and Its Clinical Significance Part II. Clin Pharmacokinet 2009; 48: 761-804.

12. Bernard S, Neville KA, Nguyen AT, Flockhart DA. Interethnic Differences in Genetic Polymorphisms of CYP2D6 in the U.S. Population: Clinical Implications. Oncologist 2006; 11: 126-35.

13. Goetz M, Knox S, Suman VJ, Rae JM, Safgren S, Ames M, et al. The impact of cytochrome P450 2D6 metabolism in women receiving adjuvant tamoxifen. Breast Cancer Res Treat 2007; 101: 113-21.

14. Schroth W, Antoniadou L, Fritz P, Schwab M, Muerdter T, Zanger UM, et al. Breast Cancer Treatment Outcome With Adjuvant Tamoxifen Relative to Patient CYP2D6 and CYP2C19 Genotypes. J Clin Oncol 2007; 25: 518793.

15. Lim HS, Ju Lee H, Seok Lee K, Sook Lee E, Jang IJ, Ro J. Clinical implications of CYP2D6 genotypes predictive of tamoxifen pharmacokinetics in metastatic breast cancer. J Clin Oncol 2007; 25: 3837-45.

16. Kiyotani K, Mushiroda T, Sasa M, Bando Y, Sumitomo I, Hosono N, et al. Impact of CYP2D6 ${ }^{\star} 10$ on recurrencefree survival in breast cancer patients receiving adjuvant tamoxifen therapy. Cancer Sci 2008; 99: 995-9.

17. Bonanni B, Macis D, Maisonneuve P, Johansson HA, Gucciardo G, Oliviero P, et al. Polymorphism in the CYP2D6 Tamoxifen-Metabolizing Gene Influences Clinical Effect but Not Hot Flashes: Data From the Italian Tamoxifen Trial. J Clin Oncol 2006; 24: 3708-9.

18. Nowell SA, Ahn J, Rae JM, Scheys JO, Trovato A, Sweeney $\mathrm{C}$, et al. Association of genetic variation in 
tamoxifen-metabolising enzyme with overall survival and recurrence of disease in brest cancer patients. Breast Cancer Res Treat 2005; 91: 249-58.

19. Wegman P, Vainikka L, Stål O, Nordenskjöld B, Skoog L, Rutqvist L-E, et al. Genotype of metabolic enzymes and the benefit of tamoxifen in postmenopausal breast cancer patients. Breast Cancer Res 2005; 7: R284-90. Disponible en http://breast-cancer-research.com/content/7/3/R284 (Consultado en noviembre 2009).

20. Wegman P, Elingarami S, Carstensen J, Stål O, Nordenskjöld B, Wingren S. Genetic variants of CYP3A5, CYP2D6, SULT1A1, UGT2B15 and tamoxifen response in postmenopausal patients with breast cancer. Breast Cancer Res 2007; 9: R7. Disponible en http://breastcancer-research.com/content/9/1/R7 (Consultado en noviembre 2009).

21. Indiana University School of Medicine, Division of clinical pharmacology. Cytochrome P450 Drug InteractionTable. Updated Nov,11, 2009 Disponible en www. drug-interactions.com (Consultado en diciembre de 2009).

22. BC Cancer Agency. Cancer Drug Manual, Tamoxifen Developed: September 1994. Revised: 1 October 2006, Limited Revision: 1 September 2008 en: http://www.bccancer.bc.ca/NR/rdonlyres/DB835 CE5-AEFA-454E-9988-AA3A239DFF2D/30169/ Tamoxifenmonograph_1August08.pdf (consultado en mayo 2010).

23. Brauch H, Jordan VC. Targeting of tamoxifen to enhance antitumour action for the treatment and prevention of breast cancer: The personalised'approach?. Eur J Cancer 2009; 45: 2274-83.

24. Fann JR, Thomas-Rich AM, Katon WJ, Cowley D, Pepping M, Mc Gregor BA, et al. Major Depression After Breast Cancer: A Review Of Epidemiology And Treatment. Gen Hosp Psych 2008; 30: 112-26.

25. Bourque F, Karama S, Looper K, Cohen V. Acute Tamoxifen-Induced Depression and Its Prevention With Venlafaxine. Psychosomatics 2009; 50: 162-5.

26. Irarrazaval ME. La depresión en el paciente oncológico. En: Repensando lo psicosomático: desde lo clínico a lo psicosocial. Ediciones de la Sociedad de Neurorologìa, Psiquiatría y Neurocirugía de Chile. Santiago: Editorial IKU; agosto 2010. p. 73-84.

27. Massie MJ, Popkin MK. Depressive Disorders. En: Holland JC editor. Psycho-oncology, New York, Oxford University Press; 1998. p. 518-40.

28. Weitzner MA, Moncello J, Jacobsen PB, Minton S. A pilot trial of paroxetine for the treatment of hot flashes and associated symptoms in women with breast cancer. J Pain Symptom Manage 2002; 23: 337-45.
29. Stearns V, Isaacs C, Rowland J, Crawford J, Ellis Mj, Kramer R, et al. A pilot trial assessing the efficacy of paroxetine hydrochloride (Paxil) in controlling hot flashes in breast cancer survivors. Ann Oncol 2000; 11: 17-22.

30. Loprinzi CL, Pisanky TM, Fonseca R, Sloan JA, Zahasky KM, Quella SK, et al. Pilot Evaluation Of Venlafaxine Hydrochloride For The Therapy Of Hot Flashes In Cancer Survivors. J Clin Oncol 1998; 16: 2377-81.

31. Loprinzi CL, Kugler JW, Sloan JA, Maillard JA, La Vasseur BI, Barton D, et al. Venlafaxine In Management Of Hot Flashes In Survivors Of Breast Cancer: A Randomized Controlled Trial. Lancet 2000; 356: 2059-63.

32. Barton D, La Vasseur B, Loprinzi C, Novotny P, Wilwerding Mb, Sloan J. Venlafaxine for the control of hot flashes: results of a longitudinal continuation study. Oncol Nurs Forum 2002; 29: 33-40.

33. Loprinzi CL, Sloan J, Stearns V, Slack R, Iyengar M, Diekmann B, et al. Newer antidepressants and gabapentin for hot flashes: an individual patient pooled analysis. J Clin Oncol 2009; 10; 27: 2831-7.

34. Ereshefsky L, Riesenman C, Lam YW. Antidepressant drug interactions and the cytochrome $\mathrm{P} 450$ system. The role of cytochrome P450 2D6. Clin Pharmacokinet 1995; 29 Suppl 1: 10-8.

35. Stearns V, Johnson Md, Rae Jm, Morocho A, Novielli A, Bhargava $\mathrm{P}$, et al. Active tamoxifen metabolite plasma concentrations after coadministration of tamoxifen and the selective serotonin reuptake inhibitor paroxetine. J Natl Cancer Inst 2003; 95: 1758-64.

36. Jin Y, Desta Z, Stearns V, Ward B, Ho H, Lee KH, et al. CYP2D6 genotype, antidepressant use, and tamoxifen metabolism during adjuvant breast cancer treatment. J Natl Cancer Inst 2005; 97: 30-9.

37. Henry L, Stearns V, Flockhart Da, Hayes D, Riba M. Drug Interactions and Pharmacogenomics in the Treatment of Breast Cancer and Depression. Am J Psychiatr 2008; 165: 10, 1251-55.

38. Desmarais Je, Looper KJ. Interactions Between Tamoxifen and Antidepressants via Cytochrome P450 2D6. J Clin Psychiatry 2009; 70: 1688-97.

39. Borges S, Desta Z, Li L, Skaar Tc, Ward Ba, Nguyen A, Jin Y. Quantitative effect of CYP2D6 genotype and inhibitors on tamoxifen metabolism: implication for optimization of breast cancer treatment. Clin Pharmacol Ther 2006; 80: 61-74.

40. Lehmann D, Nelsen J, Ramanath V, Newman N, Duggan D, Smith A. Lack of attenuation in the Antitumor Effect of Tamoxifen by Chronic CYP Isoform inhibition. J Clin Pharmacol 2004; 44: 861-5.

41. Chubak J, Buist DS, Boudreau DM, Rossing MA, Lumley $\mathrm{T}$, Weiss NS. Breast cancer recurrence risk in relation to 
antidepressant use after diagnosis. Breast Cancer Res Treat 2008; 112: 123-32.

42. Lash TL, Pedersen L, Cronin-Fenton D, Ahern TP, Rosenberg CL, Lunetta KL, et al. Tamoxifen's protection against breast cancer recurrence is not reduced by concurrent use of the SSRI citalopram. Br J Cancer 2008; 19; 99: 616-21.

43. Dezentje V, Van Blijderveen NJ, Gelderblom H, Putter H, Van Herk-Sukel MP, Casparie MK, et al. Concomitant CYP2D6 inhibitor use and tamoxifen adherence in early-stage breast cancer: A pharmacoepidemiologic study. J Clin Oncol 2009; 27: 18s (suppl; abstr CRA509).

44. Aubert RE, Stanek EJ, Yao J, Teagarden JR, Subar M, Epstein RS, et al. Comprehensive Evaluation of Tamoxifen Sequential Risk of breast cancer recurrence in women initiating tamoxifen with CYP2D6 inhibitors. J Clin Oncol 2009; 27: 18S (suppl; abstr CRA508).

45. Brauch H, Murdter TH, Eichelbaum M, Schwab M. Pharmacogenomics of Tamoxifen Therapy. C Clin Chem
2009; 55: 1770-82.

46. Higgins MJ, Rae JM, Flockhart DA, Hayes DF, Stearns V. Pharmacogenetics of tamoxifen: who should undergo CYP2D6 genetic testing?. J Natl Compr Canc Netw 2009; 7: 203-13.

47. Hoskins JM, Carey LA, Mc Leod HL. CYP2D6 and tamoxifen: DNA matters in breast cancer. Nat Rev Cancer 2009; 9: 576-86.

48. Goetz MP, Kamal A, Ames MM. Tamoxifen pharmacogenomics: the role of CYP2D6 as a predictor of drug response. Clin Pharmacol Ther 2008; 83: 160-6.

49. Rock CL, Demark-Wahnefried W. Nutrition and survival after the diagnosis of breast cancer: A review of the evidence. J Clin Oncol 2002; 20: 3302-16.

50. Mc Tiernan A, Irwin M, Von Gruenigen V. Weight, Physical Activity, Diet, and Prognosis in Breast and Gynecologic Cancers. J Clin Oncol 2010; Jul 19 en: http:// jco.ascopubs.org/cgi/doi/10.1200/JCO.2010.27.9752 (consultado en agosto 2010). 